\title{
COVID-19, Police Violence, and Educational Disruption:The Differential Experience of Anxiety for Racial and Ethnic Households
}

\author{
Ashley E. Burch ${ }^{1} \cdot$ Molly Jacobs ${ }^{1}$
}

Received: 16 August 2021 / Revised: 4 November 2021 / Accepted: 5 November 2021 / Published online: 1 December 2021

(c) W. Montague Cobb-NMA Health Institute 2021

\begin{abstract}
Background The threat of a deadly pandemic, racial tension, recessionary economic circumstances, and educational disruption likely contributed to the heightened anxiety felt by many Americans in 2020. This study examines the differential anxiety experienced by Black, White, and Hispanic households with and without children during 2020.

Method Data from the Census Bureau's Household Pulse Survey detailing the frequency of anxiety among a nationally representative sample of adults from April 23 to December 21, 2020, was coupled with Centers for Disease Control and Prevention records of COVID-19 diagnoses and state-level police killings. Multinomial logistic regression assessed the relative contribution of COVID-19 deaths, police violence, unemployment, fear of unemployment, change in educational delivery, and geographic location to anxiety among racial/ethnic cohorts with and without children.

Results Anxiety frequency increased over the sample for all groups. However, White anxiety was highly responsive to statelevel COVID-19 fatalities, while Black anxiety was highly correlated with police violence. Households with children showed higher levels of anxiety during nontraditional educational delivery, whereas both households with and without children experienced high levels of fear regarding employment uncertainty and poverty.

Conclusions Experiences in 2020 impacted all groups differently, but each showed a high frequency of anxiety.
\end{abstract}

Keywords COVID $\cdot$ Police violence $\cdot$ Educational disruption $\cdot$ Black $\cdot$ Anxiety

\section{Introduction}

The year 2020 ushered in an unprecedented level of worldwide disruption and uncertainty as the coronavirus disease 2019 (COVID-19) spread across the globe [1]. The extensive reach of the pandemic impacted nearly every facet of society resulting in economic instability, pervasive illness and mortality, and social disruption [2-6]. Nationally, the uncertainty of 2020 was compounded for people in the USA as they experienced political divisiveness and racial unrest comprised of protests, riots, and police brutality [7-9]. Despite the nationwide uncertainty that was shared by virtually every American, inequality in the economic and health effects of the pandemic has been observed for those of different racial and ethnic groups [10-13]. To characterize the

Ashley E. Burch

burchas15@ecu.edu

1 Department of Health Services and Information Management, East Carolina University, 4340N Health

Sciences Building, Greenville, NC 27858, USA uncertainty experienced in 2020, we must identify overlapping and unique factors related to anxiety reported within different racial and ethnic groups.

Early in the COVID-19 pandemic, misinformation and rapidly evolving public health guidelines were a source of insecurity for many Americans [14, 15]. Political leaders and scientists contributed to the confusion by providing, at times, conflicting information on the severity of the virus, the necessity of reducing transmission, infection risk, and treatment [16-18]. As COVID-19 reached pandemic proportions, individuals vulnerable to mental illness experienced an increase in post-traumatic stress symptoms, depression, sleep disturbances, substance use, and attempted suicide [19-24]. Even among adults without a history of mental health conditions, the trauma inflicted by the pandemic has led to an increase in psychological distress. A survey of 9687 adults participating in the Pew Research Center's American Trends Panel found that more than a quarter of respondents suffered distress in the form of anxiety, sleep disturbance, depression, or hyperarousal [25]. Pre-pandemic circumstances, including economic and social support, as well as 
individual factors such as race, ethnicity, sex, and age, are likely to influence the level of anxiety experienced as a result of COVID-19. Studies have found being female, White nonHispanic, reporting financial uncertainty, and loneliness are risk factors associated with greater mental distress, while those who are older, employed, or of Asian American or Hispanic/Latino descent were less likely to report pandemicrelated mental distress [25-30].

Extensive media coverage of police violence and subsequent social unrest was another source of heightened anxiety during 2020 [31, 32]. Contact with police, specifically police-related violence, is disproportionately experienced by minority communities. In the USA, police shootings of unarmed Black residents are 3.5-fold greater than shootings of unarmed White residents [33]. Among individuals associated with a police-involved fatality between 2012 and 2018, Black men had an estimated risk between 1.9 and 2.4 deaths per 100,000 annually while White men had an estimated risk between 0.6 and 0.7 with the risk for Hispanic men falling in between (0.8-1.2) [34]. Although the majority of policeinvolved fatalities result in the death of an adult, minority children residing close to the location of violence are absent from school in the days following the killing and experience decreases in educational attainment [35]. Given the detrimental impact of police violence on children [36], caregivers living in proximity to police-related killings that occurred during 2020 may be at an increased risk for anxiety.

An additional source of anxiety for households with children was the extensive school closures intended to keep students safe from the spread of COVID-19. Over the course of 2020, the United Nations Educational, Scientific and Cultural Organization estimated that 1.6 billion students globally experienced educational disruptions [37]. As schools shut down for an average of 3.5 months in response to isolation mandates, remote learning was adopted by many school districts, leaving caregivers to become pseudo-educators [38]. The success of remote learning is largely dependent on access to technology and caregiver involvement. Computer ownership, a significant factor in the so-called digital divide, is more likely in households where residents were White, employed, and lived above the poverty level [39, 40]. Furthermore, Americans living in rural areas and those in racial/ethnic minority groups are disproportionately represented among the approximately 18 million people in the USA who lack Internet access [41-44]. Attempting to manage the educational needs of children, while lacking the resources to facilitate remote learning, could be a reason for the significantly higher levels of stress seen among adults with children during the pandemic compared to those without children [45]. Lee and colleagues found that parents' perceived ability to provide at-home education, including access to resources, was related to increased depression and stress [46].
Notwithstanding the novel stress brought on directly by COVID-19, namely, a global public health crisis, the pandemic also magnified and, in some instances, compounded other adverse life events. Over the last year, loss of employment, job insecurity, and poverty were significant sources of stress for many Americans [30, 47, 48]. Although racial inequality in employment is not novel $[49,50]$, the COVID19 pandemic has intensified the divide, leaving minority communities to disproportionately endure the burden of unemployment. Early in the pandemic, Blacks experienced an average unemployment rate of $16.6 \%$ compared to $12.8 \%$ for Whites; yet, the highest level of unemployment during this time was seen for Hispanics (18.2\%) [11]. For Whites and Hispanics, subsequent months brought some recovery with unemployment declining by 3.6 and $8.3 \%$ points, respectively. However, among Blacks, unemployment fell by less than $2 \%(1.5 \%)$ [11]. Likewise, drastic increases are reported in the representation of Blacks and Hispanics (odds ratios of 1.64 and 2.84, respectively) living in severe poverty-defined as surviving on less than $\$ 2.00$ per day [51, 52].

The current study examined the effects of various stressors including COVID-19 mortality, police violence, and economic disruption that may result in a differential effect in the experience of anxiety among Blacks, Whites, and Hispanics. In addition, we investigate households with and without children to further extrapolate the effect of education disruption on the level of anxiety experienced during 2020.

\section{Methods}

\section{Data}

\section{HHPS}

Data from this study was drawn from the first 21 weeks of the Household Pulse Survey (HHPS) — a quick deployment data collection instrument designed to collect data on a range of ways in which people's lives have been impacted by the COVID-19 pandemic [53]. The result of a collaboration between the US Census Bureau and other federal agencies, the HHPS asked individuals about their experiences in terms of employment status, spending patterns, food security, housing, physical and mental health, access to health care, and educational disruption. The HHPS was conducted in three phases. Phase 1, which began on April 23, 2020, asked individuals about their experiences in terms of employment status, food security, housing, physical and mental health, access to health care, and educational disruption. Phases 2 and 3, beginning on August 19 and October 28,2020 , respectively, carried over many of these questions to allow users to understand how these domains changed as 
the pandemic continued. The instrument utilized an overlapping weekly panel of respondents, each of whom was surveyed once per week for 3 consecutive weeks before being replaced by a new panel [53].

During the collection period, the Census Bureau sampled nearly 1.9 million and 1.0 million housing units in weeks 1 and 2 of the survey, respectively. An additional 1.1 million addresses were added each week thereafter to ensure that the survey remained representative of the US population. In total, the survey sampled approximately 13.8 million housing units collecting roughly 108,000 responses per week, for an approximate 5.0\% response rate. Households were surveyed on basic demographic information including size, the birth year of the responding adult, sex, race, and ethnicity. While race, ethnicity, sex, and age were collected to ensure a representative sample, the educational attainment of the responding adult was also collected to align the final weighted distribution with the 2018 American Community Survey estimates of the adult population's educational attainment.

While some content varied between the three phases of the HHPS, this study utilized an instrument to measure anxiety that was present throughout the panel. Respondents indicated, "Over the last 7 days, how often have you been bothered by the following problems: feeling nervous, anxious, or on edge? Would you say not at all, several days, more than half the days, or nearly every day?" Response frequencies were translated into ordered response categories indicating frequency such that $0=$ not at all, $1=$ several days, $2=$ more than half the days, and $3=$ nearly every day. To capture labor market effects related to the pandemic, HHPS respondents were asked two labor market-related questions (referred to as "job loss" and "fear of job loss," respectively):

Have you or has anyone in your household experienced a loss of employment income since March 13, 2020?

Do you expect that you or anyone in your household will experience a loss of employment income in the next 4 weeks because of the coronavirus pandemic?

Household respondents indicate whether any child in the household was enrolled in a public school, enrolled in a private school, or educated in a homeschool setting in kindergarten through 12th grade or grade equivalent at any time during the 2020-2021 school year. Those households who responded affirmatively were then asked "How has the coronavirus pandemic affected how the children in this household received education for the 2020-2021 school year? Response choices included the following: classes normally taught in person at the school were canceled; classes normally taught in person moved to a distance-learning format using online resources, either self-paced or in real time; classes normally taught in person moved to a distancelearning format using paper materials sent home to children; classes normally taught in person changed in some other way; and there was no change because schools did not close. These survey items were used to create a binary indicator which assumed a value of one if the educational delivery format changed in any way and zero otherwise.

Finally, respondents classified their 2019 pretax income into one of eight categories: (1) less than $\$ 25,000$, (2) $\$ 25,000-\$ 34,999$, (3) $\$ 35,000-\$ 49,999$, (4) $\$ 50,000-\$ 74,999$, (5) $\$ 75,000-\$ 99,999$, (6) $\$ 100,000-\$ 149,999$, (7) $\$ 150,000-\$ 199,999$, and (8) $\$ 200,000$ and above. These categories along with the reported number of individuals residing in the household were used to create two indicators-poverty and low income. Poverty indicates households reported income below $100 \%$ of the 2020 Health and Human Services (HHS) Poverty earnings threshold for their household size. Low income indicates household reported income below $150 \%$ of the HHS Poverty earning threshold for their household size.

\section{MPV Database}

Racial tension during 2020 was prompted by several notable African American deaths at the hands of law enforcement. While it is not possible to account for the emotional, psychological, and social impact of these tragedies, this study includes the total number of police killings in each state during each week of the HHPS. Data was derived from the Mapping Police Violence (MPV) database. MPV sources information from three large, comprehensive databases on police killings: FatalEncounters.org, the US Police Shootings Database, and KilledbyPolice.net. In addition to compiling the information from these sources, MPV verifies data quality and completeness through verification by media outlets, obituaries, criminal records databases, and police reports and, when possible, includes additional information such as race, vehicular use, and armament status. Compared to other sources of police killings, such as the Washington Post Fatal Encounters database, MPV includes deaths that result from chokeholds, batons, tasers, or other means. This study matches respondents' state of residence and week of participating in the HHPS with the total number of police killings in their state during that time.

\section{COVID-19 Deaths}

To capture the severity of the COVID-19 pandemic in the respondents' local areas, COVID-19 Case Surveillance Public Use Data was obtained from the Centers for Disease Control and Prevention (CDC). The CDC recorded and archived daily numbers of confirmed cases and confirmed deaths for each state throughout the COVID-19 pandemic. Since each week of the HPS survey was conducted over a range of days, daily values for COVID-19 cases corresponding to the dates 
of the survey week are averaged to calculate the state-level weekly average confirmed new COVID-19 deaths for inclusion in the regression model.

\section{Empirical Estimation}

Ordered logistic (OL) regression evaluated those factors related to anxiety among Blacks, Whites, and Hispanics during the 2020 COVID-19 pandemic. Anxiety was expressed as a discrete, numeric category value representing the frequency of anxiety (coded such that $0=$ not at all, $1=$ several days, $2=$ more than half the days, and $3=$ nearly every day). Dichotomous variables for the female sex, residing in a metropolitan statistical area (MSA), job loss, fear of job loss, poverty, and low income were included in the model. Age, week of the HHPS (ranging from 1 to 21), and the total number of people residing in the household were also added. To account for anxiety related to the pandemic and police violence, the total number of confirmed, new COVID-19 cases, and the total number of police killings for each state and week were also included. Finally, a binary variable for HHPS week 5 (May 28-June 2)-the week directly following the death of George Floyd-was included as an indicator of social unrest.

As those factors related to anxiety will likely vary between households with and without school-aged children, separate regression models were run for households containing individuals under age 18. Additionally, an indicator for those experiencing a change in learning format (i.e., classes normally taught in person were delivered via an alternative means) was included for households with children. Finally, separate regression models were run for Black, White, and Hispanic households to allow for variations among those factors related to anxiety frequency. To assess household variability among vulnerable populations, we also ran a full sample regression with racial and ethnic interaction terms.

\section{Robustness Test}

While separate regression models for Black, White, and Hispanic households provide easily interpretable estimates, similar anxiety among these racial and ethnic groups can also be estimated simultaneously. The inclusion of binary indicators for Black and Hispanic household tests for differences in the average anxiety level experienced by these groups over the sample period. To test the differential contribution of various elements to the anxiety experienced by these groups, interaction terms are also included between these racial/ethnic indicators and those factors hypothesized to be related to anxiety. An ordered logistic regression including these binary indicators and interactions is run to test the robustness of the subgroup models described above.

\section{Results}

\section{Respondent Characteristics: Households with Children}

Sample Characteristics Table 1 lists sample characteristics for Black, White, and Hispanic households with children (HHWC). On average, 60 to $75 \%$ of HHWC had a female between 40 and 45 years of age responding to the HHPS. These households have between 4 and 5 members. However, only about $25 \%$ of Whites live in MSAs compared to 43.21 and $45.75 \%$ of Blacks and Hispanics, respectively-a statistically significant difference $\left(\chi^{2}=13,130.3773, p<0.0001\right)$. Similarly, more Blacks (85.12\%) and Hispanics $(89.56 \%)$ live in the South than White (75.18) $\left(\chi^{2}=6487.0006\right.$, $p<0.0001)$. Over $40 \%$ of Hispanics and Blacks report that they expect to lose their job due to the pandemic, compared to only $26 \%$ of Whites $\left(\chi^{2}=7498.0560<0.0001\right)$. Fewer Whites (43.66\%) than Blacks (54.96\%) or Hispanics $(57.09 \%)$ report that someone in their household has lost their job as a result of pandemic-related economic shifts. Likely related to job loss or insecurity, a smaller proportion of White households earn at the poverty $(4.1 \%)$ or low-income threshold (21.1\%) compared to Black (poverty: 13.9\%, low-income: $49.49 \%$ ) and Hispanic households (poverty: $8.53 \%$, low-income: $43.90 \%$ ) (poverty: $\chi^{2}=7163.3303$, $p<0.0001$; low-income: $\left(\chi^{2}=23,183.3478, p<0.0001\right)$. However, similar percentages of White $(94.50 \%)$, Black (97.52\%), and Hispanic (96.76\%) households with children were forced to change learning formats because of school closures/scheduling changes. But differences were statistically significant $\left(\chi^{2}=1006.6270, p<0.0001\right)$. While Blacks, Whites, and Hispanics report roughly similar percentages of anxiety frequencies; they do appear to differ statistically $\left(\chi^{2}=428.0222, p<0.0001\right)$.

\section{Respondent Characteristics: Households Without Children}

Sample Characteristics Table 2 lists sample characteristics for Black, White, and Hispanic households without children (HHWOC). All households are comprised of about two people. A significantly larger proportion of Black (65.89\%) household respondents are female compared to White $(56.75 \%)$ or Hispanic $(56.23 \%)$ household respondents $\left(\chi^{2}=2409.3543, p<0.0001\right)$. White household respondents were significantly older (57.09) than Black (52.23) or Hispanic (49.49) respondents $(t$-stat $=4242.30, p<0.0001)$. Much like HHWC, a larger proportion of Hispanic (46.22\%) and Black (47.93\%) HHWOC reside in MSAs than White 


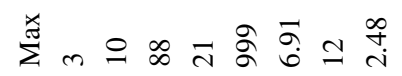

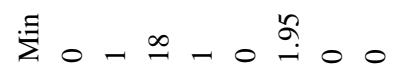

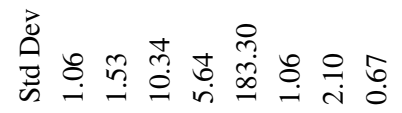

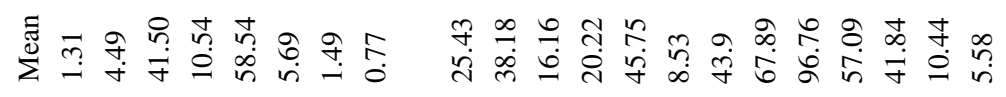

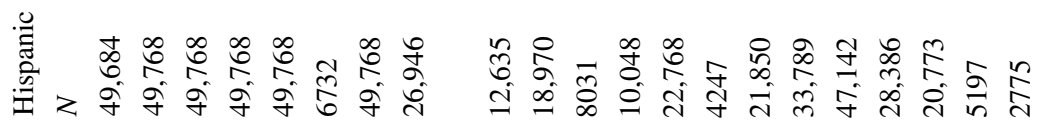

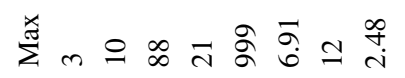

紊 $0-\stackrel{\infty}{-}$ - $\stackrel{n}{=} 00$

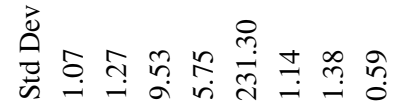

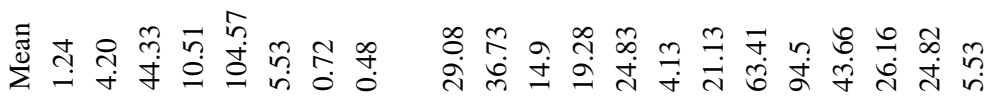

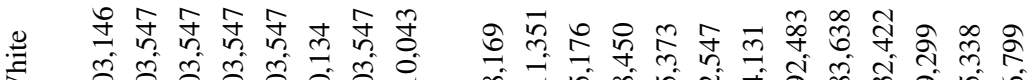

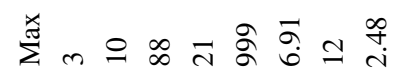

竞 $0-\stackrel{\infty}{\rightarrow}-\stackrel{n}{=} 00$

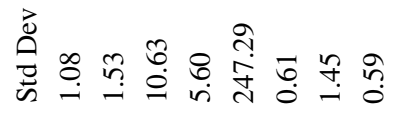

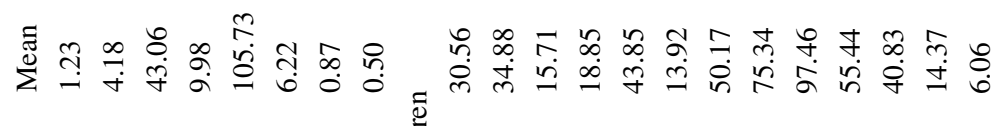

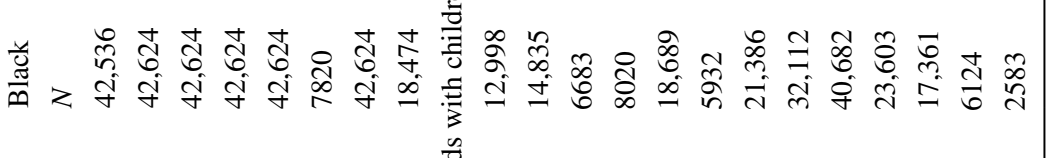

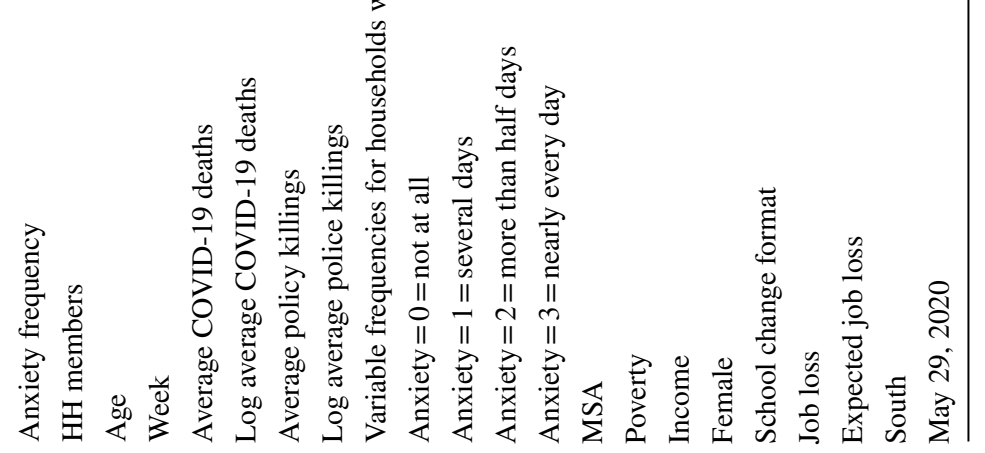




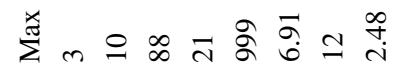

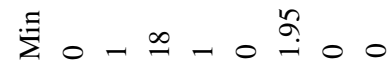

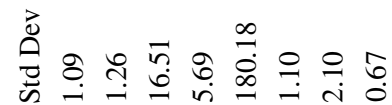

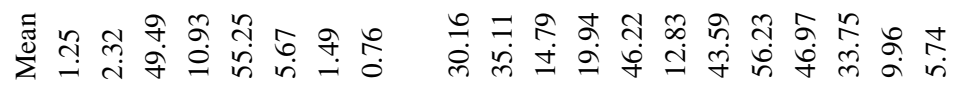

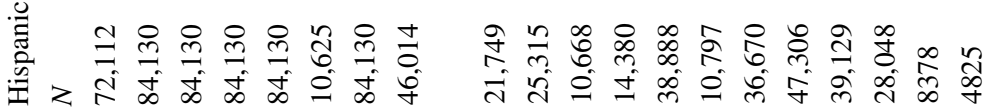

尊n

往

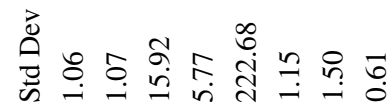

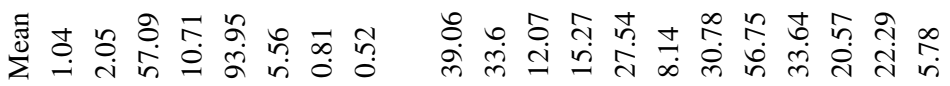

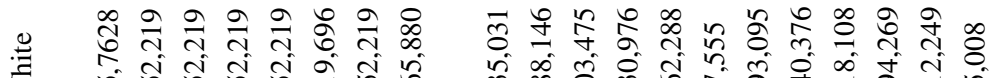

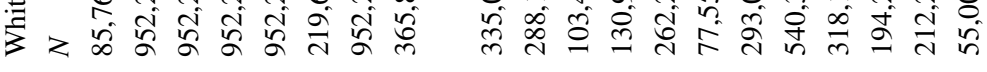

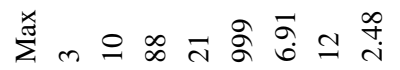

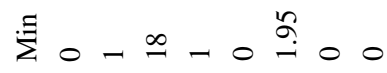

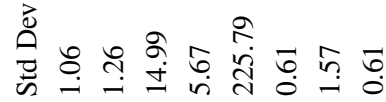

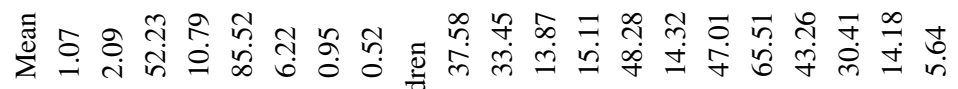

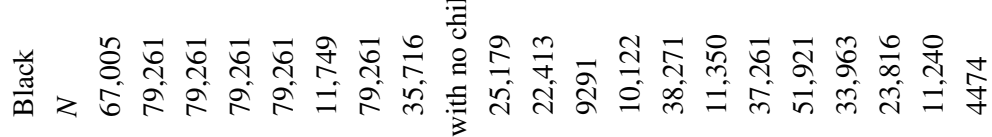

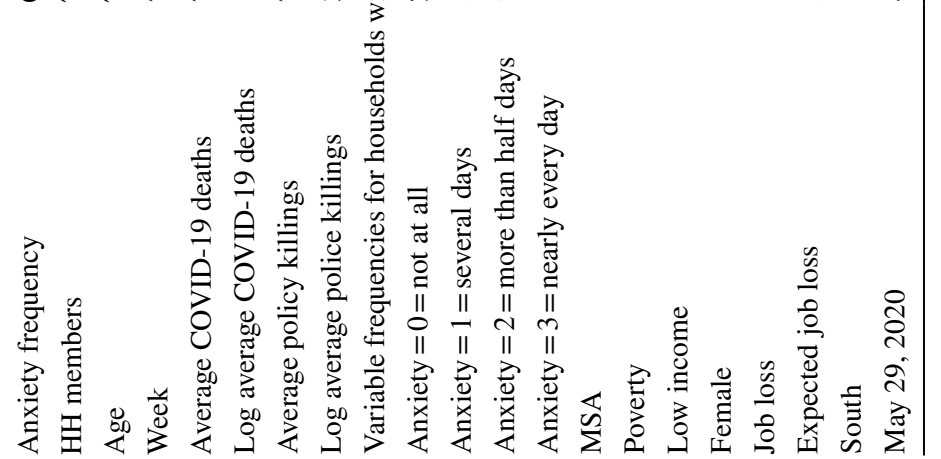


(27.54\%) HHWOC $\left(\chi^{2}=24,513.7412, p<0.0001\right)$. However, significantly more White HHWOC $(22.29 \%)$ reside in the South than Black $(14.45 \%)$ or Hispanic $(9.96 \%)$ HHWOC $\left(\chi^{2}=9105.3969, p<0.0001\right)$. Similar to the proportions described above, more Blacks and Hispanics have lost their jobs $\left(\chi^{2}=7932.6860, p<0.0001\right)$ or fear that they will lose their jobs $\left(\chi^{2}=10,456.7296, p<0.0001\right)$ as a result of the pandemic than Whites. Fewer White (8.14\%) HHWOC earn at or below the poverty threshold than Black $(14.19 \%)$ or Hispanic $(12.83 \%)\left(\chi^{2}=4853.8872, p<0.0001\right)$ and similar trends are observed among low-income earners (White: 30.78, Black: 46.59, Hispanic: $43.59 ; \chi^{2}=12,648.9586$, $p<0.0001)$. While reported anxiety frequency differs significantly between the three groups $\left(\chi^{2}=2891.3814\right.$, $p<0.0001$ ), those factors most closely related to fear among the subgroups can only be compared with multivariate statistics.

\section{Regression: Households with Children}

Regression Analysis Regression results, odds ratios, and confidence intervals are listed in Table 3 for HHWC separately by subgroup. Estimates in an ordered logistic regression indicate that for a one-unit increase in the predictor, the response variable level is expected to change by its respective regression coefficient in the ordered log-odds scale while the other variables in the model are held constant. Odds ratios provide a more intuitive interpretation indicating the odds of a higher-ordered frequency of anxiety given a change in the independent regressor, given the other variables are held constant. All subgroups had an odds ratio greater than one for a week suggesting higher odds of more frequent anxiety as the pandemic progressed (OR: Black: 1.049, White: 1.035, Hispanic: 1.009). Similarly, higher age is associated with a lower frequency of anxiety (OR: Black: 0.984, White: 0.984, Hispanic: 0.99). As expected, having recently lost a job (OR: Black: 1.352, White: 1.423, Hispanic: 1.376) or expecting to lose a job (OR: Black: 1.53, White: 2.076, Hispanic: 2.014) is associated with significantly more frequent anxiety. Households earning below the poverty threshold experience higher odds of frequent anxiety (OR: Black: 1.413, White: 1.577, Hispanic: 1.067). However, only Blacks (OR: 1.042) and Whites (OR: 1.143) show a positive relationship between anxiety and low income.

Shifting to an alternative learning format increases the odds of higher anxiety for Blacks $(\mathrm{OR}=2.67)$ and Whites $(\mathrm{OR}=2.096)$ but has a slightly negative effect for Hispanics $(\mathrm{OR}=0.875)$. Interestingly, the number of newly reported COVID-19 cases is only associated with higher anxiety among Whites $(\mathrm{OR}=1.044)$. However, all three subgroups
(OR: Black: 1.303, White: 1.158, Hispanic: 1.065) show a higher likelihood of more frequent anxiety associated with higher levels of police killings with the largest magnitude seem among Blacks. Similarly, May 29, 2020-the week following the publicized killing of George Floyd-was associated with a $47 \%$ increase in the odds of frequency anxiety among Blacks and an 11\% increase among Whites.

\section{Regression: Households Without Children}

Regression Analysis Regression results, odds ratios, and confidence intervals are listed in Table 4 for HHWOC separately by subgroup. Odds of higher anxiety among HHWOC are observed among those living in MSAs (OR: Black: 1.363, White: 1.024, Hispanic: 1.079), residing in larger households (OR: Black: 1.147, White: 1.016, Hispanic: 1.002), and earning low (OR: Black: 1.288, White: 1.104, Hispanic: 1.145) or poverty-level income (OR: Black: 1.135, White: 1.814, Hispanic: 1.045). Households with female respondents have higher odds of frequent anxiety (OR: Black: 1.428, White: 1.816, Hispanic: 1.9 ) than those with male respondents. For Blacks $(\mathrm{OR}=1.165)$ and Hispanics ( $\mathrm{OR}=1.055)$, residing in the South increases the likelihood of frequent anxiety but is associated with lower anxiety frequency for Whites ( $\mathrm{OR}=0.854)$. As observed among HHWC, later weeks of the HHPS have higher odds of anxiety (OR: Black: 1.041, White: 1.027, Hispanic: 1.023). Results for job loss (OR: Black: 1.771, White: 1.388, Hispanic: 1.402) or expected job loss (OR: Black: 1.286, White: 1.872, Hispanic: 1.847) are similar to those seen in HHWC.

COVID-19 cases were associated with higher anxiety in Whites $(\mathrm{OR}=1.049)$ and Hispanics $(\mathrm{OR}=1.01)$, but not in Blacks $(\mathrm{OR}=0.675)$. Police killings, however, were associated with $64 \%$ higher anxiety in Blacks, $39.4 \%$ in Hispanics, and $4.5 \%$ in Whites. The week following the killing of George Floyd was also associated with similarly high anxiety in Blacks and Hispanics, and very little change in the anxiety of Whites, compared to other times during 2020 (OR: Blacks: 1.452, Whites: 0.973, Hispanics: 1.124).

\section{Regression: Full Sample Regression with Racial/ Ethnic Interactions}

Results from the full sample regression with racial and ethnic interaction terms are listed in Appendix 1 Table 5. The significance and magnitude of most covariates are similar to previous results. The additional interaction terms are 
Table 3 Regression results for Black, White, and Hispanic households with children

Ordered logistic regression model for households with children

\begin{tabular}{|c|c|c|c|c|c|c|c|c|c|}
\hline & \multicolumn{3}{|l|}{ Black } & \multicolumn{3}{|l|}{ White } & \multicolumn{3}{|l|}{ Hispanic } \\
\hline & \multicolumn{9}{|c|}{ Model fit statistics } \\
\hline AIC & \multicolumn{3}{|l|}{$21,184,492$} & \multicolumn{3}{|l|}{$88,721,738$} & \multicolumn{3}{|l|}{$15,770,690$} \\
\hline $\mathrm{SC}$ & \multicolumn{3}{|l|}{$21,184,509$} & \multicolumn{3}{|l|}{$88,721,761$} & \multicolumn{3}{|l|}{$15,770,706$} \\
\hline \multirow[t]{3}{*}{$-2 \log L$} & $21,184,486$ & & & $88,721,732$ & & & $15,770,684$ & & \\
\hline & \multicolumn{9}{|c|}{ Maximum likelihood estimates } \\
\hline & Estimate & Std err & Chi squ & Estimate & Std err & Chi squ & Estimate & Std err & Chi squ \\
\hline Intercept (3) & -1.4288 & 0.0143 & $10,018.54$ & -2.9087 & 0.00418 & $483,884.8$ & -0.8599 & 0.0119 & 5253.335 \\
\hline Intercept (2) & -0.3913 & 0.0143 & 752.5929 & -2.0647 & 0.00417 & $245,520.8$ & 0.2167 & 0.0119 & 334.2517 \\
\hline Intercept (1) & 1.1017 & 0.0143 & 5958.552 & -0.3866 & 0.00415 & 8674.624 & 1.7648 & 0.0119 & $22,093.39$ \\
\hline HH size & -0.0392 & 0.000433 & 8181.696 & -0.00329 & 0.00024 & 188.2153 & 0.0868 & 0.000517 & $28,169.19$ \\
\hline MSA & 0.1875 & 0.00235 & 6371.553 & 0.2603 & 0.00144 & $32,607.9$ & -0.2359 & 0.00218 & $11,748.56$ \\
\hline South & -0.0376 & 0.00296 & 160.7103 & -0.2605 & 0.000945 & $75,970.79$ & -0.5009 & 0.00281 & $31,805.37$ \\
\hline Poverty & 0.3459 & 0.0022 & $24,701.84$ & 0.4555 & 0.0015 & $92,076.62$ & 0.0645 & 0.00318 & 412.2512 \\
\hline Low income & 0.0415 & 0.00162 & 656.2494 & 0.1337 & 0.000792 & $28,513.9$ & -0.1781 & 0.00175 & $10,342.68$ \\
\hline Female & 0.2307 & 0.0014 & $27,131.99$ & 0.5859 & 0.000655 & $799,312.1$ & 0.7231 & 0.00157 & $211,340.4$ \\
\hline Age & -0.0156 & 0.000059 & $70,245.63$ & -0.0179 & 0.000029 & $387,124.9$ & -0.00971 & 0.000067 & $21,064.86$ \\
\hline Week & 0.0481 & 0.000196 & $59,967.28$ & 0.0347 & 0.000068 & $258,416.2$ & 0.00919 & 0.000166 & 3060.171 \\
\hline School change & 0.982 & 0.00886 & $12,272.89$ & 0.7401 & 0.00212 & $121,787.2$ & $-\mathbf{0 . 1 3 3 3}$ & 0.0058 & 528.0172 \\
\hline logCOVID-19 deaths & -0.2485 & 0.00188 & $17,439.63$ & 0.0427 & 0.000548 & 6066.987 & -0.2615 & 0.00163 & $25,862.85$ \\
\hline logPoliceKillings & 0.2646 & 0.0017 & $24,251.33$ & 0.1471 & 0.000864 & $28,998.91$ & 0.0631 & 0.00201 & 989.1421 \\
\hline Lost job & 0.3017 & 0.00154 & $38,200.03$ & 0.3524 & 0.000762 & $214,110.5$ & 0.3188 & 0.00196 & $26,552.25$ \\
\hline Expect to lose Job & 0.4252 & 0.00153 & $77,171.84$ & 0.7305 & 0.000813 & $807,614.3$ & 0.7001 & 0.0019 & $135,665.3$ \\
\hline \multirow[t]{3}{*}{ May 29, 2020} & 0.3899 & 0.00381 & $10,449.19$ & 0.1051 & 0.00133 & 6294.337 & -0.8783 & 0.00384 & $52,224.11$ \\
\hline & \multicolumn{9}{|l|}{ Odds ratios } \\
\hline & Estimate & $95 \% \mathrm{CL}$ & & Estimate & $95 \% \mathrm{CL}$ & & Estimate & $95 \% \mathrm{CL}$ & \\
\hline $\mathrm{HH}$ size & 0.962 & 0.961 & 0.962 & 0.997 & 0.996 & 0.997 & 1.091 & 1.09 & 1.092 \\
\hline MSA & 1.206 & 1.201 & 1.212 & 1.297 & 1.294 & 1.301 & 0.79 & 0.787 & 0.793 \\
\hline South & 0.963 & 0.958 & 0.969 & 0.771 & 0.769 & 0.772 & 0.606 & 0.603 & 0.609 \\
\hline Poverty & 1.413 & 1.407 & 1.419 & 1.577 & 1.572 & 1.582 & 1.067 & 1.06 & 1.073 \\
\hline Low income & 1.042 & 1.039 & 1.046 & 1.143 & 1.141 & 1.145 & 0.837 & 0.834 & 0.84 \\
\hline Female & 1.259 & 1.256 & 1.263 & 1.797 & 1.794 & 1.799 & 2.061 & 2.054 & 2.067 \\
\hline Age & 0.984 & 0.984 & 0.985 & 0.982 & 0.982 & 0.982 & 0.99 & 0.99 & 0.99 \\
\hline Week & 1.049 & 1.049 & 1.05 & 1.035 & 1.035 & 1.035 & 1.009 & 1.009 & 1.01 \\
\hline School change & 2.67 & 2.624 & 2.717 & 2.096 & 2.087 & 2.105 & 0.875 & 0.865 & 0.885 \\
\hline $\log$ COVID-19 deaths & 0.78 & 0.777 & 0.783 & 1.044 & 1.043 & 1.045 & 0.77 & 0.767 & 0.772 \\
\hline logPolice killings & 1.303 & 1.299 & 1.307 & 1.158 & 1.157 & 1.16 & 1.065 & 1.061 & 1.069 \\
\hline Lost job & 1.352 & 1.348 & 1.356 & 1.423 & 1.42 & 1.425 & 1.376 & 1.37 & 1.381 \\
\hline Expect to lose job & 1.53 & 1.525 & 1.534 & 2.076 & 2.073 & 2.079 & 2.014 & 2.006 & 2.021 \\
\hline May 29, 2020 & 1.477 & 1.466 & 1.488 & 1.111 & 1.108 & 1.114 & 0.415 & 0.412 & 0.419 \\
\hline
\end{tabular}

Significant at $95 \%$ confidence level

Dependent variable: frequency of anxiety $(0=$ not at all, $1=$ several days, $2=$ more than half the days, $3=$ nearly every day $)$

Estimates weight by person and household

statistically significant, but the coefficients cannot be interpreted directly given the ordered nature of the dependent variable. Instead, they indicate that anxiety within racial and ethnic households of various sizes and at various socioeconomic levels displays some variations depending on its relative composition. 
Table 4 Regression results for Black, White, and Hispanic households without children

\begin{tabular}{|c|c|c|c|c|c|c|c|c|c|}
\hline \multicolumn{10}{|c|}{ Ordered logistic regression model for households with no children } \\
\hline & \multicolumn{3}{|l|}{ Black } & \multicolumn{3}{|l|}{ White } & \multicolumn{3}{|l|}{ Hispanic } \\
\hline & \multicolumn{9}{|c|}{ Model fit statistics } \\
\hline AIC & \multicolumn{3}{|l|}{$25,994,242$} & \multicolumn{3}{|l|}{$239,691,564$} & \multicolumn{3}{|l|}{$19,382,969$} \\
\hline $\mathrm{SC}$ & \multicolumn{3}{|l|}{$25,994,260$} & \multicolumn{3}{|c|}{$239,691,591$} & \multicolumn{3}{|l|}{$19,382,986$} \\
\hline \multirow[t]{3}{*}{$-2 \log L$} & $25,994,236$ & & & $239,691,55$ & & & $19,382,963$ & & \\
\hline & \multicolumn{9}{|c|}{ Maximum likelihood estimates } \\
\hline & Estimate & Std err & Wald & Estimate & Std err & Wald & Estimate & Std err & Wald \\
\hline Intercept (3) & -0.3309 & 0.0108 & 942.6386 & -1.6659 & 0.00208 & $641,699.2$ & -2.0426 & 0.00799 & $65,370.14$ \\
\hline Intercept (2) & 0.4914 & 0.0108 & 2080.422 & -0.8435 & 0.00207 & $165,669.6$ & -1.4336 & 0.00797 & $32,353.25$ \\
\hline Intercept (1) & 2.0137 & 0.0108 & $34,825.22$ & 0.733 & 0.00207 & $125,122.3$ & 0.223 & 0.00795 & 786.3544 \\
\hline HH size & 0.1373 & 0.000296 & $214,687.4$ & 0.016 & 0.00012 & $17,663.08$ & 0.0015 & 0.000387 & 15.0127 \\
\hline MSA & 0.3095 & 0.00209 & 21,921 & 0.0238 & 0.000881 & 727.5656 & 0.0758 & 0.00194 & 1526.431 \\
\hline South & 0.1529 & 0.00276 & 3066.706 & -0.1578 & 0.000582 & $73,532.46$ & 0.0534 & 0.00295 & 326.4658 \\
\hline Poverty & 0.1267 & 0.00174 & 5329.267 & 0.5956 & 0.000717 & $689,434.9$ & 0.0439 & 0.00229 & 368.5271 \\
\hline Low income & 0.2534 & 0.0015 & $28,631.79$ & 0.0985 & 0.000524 & $35,384.75$ & 0.135 & 0.00171 & 6262.711 \\
\hline Female & 0.3564 & 0.00121 & $87,275.99$ & 0.5965 & 0.000393 & $2,303,044$ & 0.6419 & 0.00139 & $212,214.2$ \\
\hline Age & -0.0124 & 0.000039 & $101,929.1$ & -0.0268 & 0.000012 & $5,400,532$ & -0.0112 & 0.00004 & $77,472.9$ \\
\hline Week & 0.0405 & 0.000165 & $60,348.07$ & 0.0271 & 0.00004 & $460,553.1$ & 0.023 & 0.000141 & $26,638.51$ \\
\hline logCOVID-19 deaths & -0.3928 & 0.00171 & $52,946.89$ & 0.0477 & 0.000327 & $21,365.46$ & 0.00953 & 0.00127 & 56.6654 \\
\hline logPolice killings & 0.4974 & 0.00155 & $102,880.9$ & 0.0438 & 0.000531 & 6789.953 & 0.332 & 0.00181 & $33,491.22$ \\
\hline Lost Job & 0.5715 & 0.00146 & $152,540.2$ & 0.3279 & 0.000511 & $412,344.3$ & 0.3376 & 0.00167 & $41,041.54$ \\
\hline Expect to lose job & 0.2439 & 0.00143 & 28,954 & 0.6268 & 0.000554 & $1,281,327$ & 0.6136 & 0.0017 & 130,868 \\
\hline \multirow[t]{3}{*}{ May 29, 2020} & 0.373 & 0.0049 & 5794.925 & $-\mathbf{0 . 0 2 7 3}$ & 0.000783 & 1216.679 & 0.1165 & 0.00314 & 1374.721 \\
\hline & \multicolumn{9}{|l|}{ Odds ratios } \\
\hline & Estimate & $95 \% \mathrm{CL}$ & & Estimate & $95 \% \mathrm{CL}$ & & Estimate & $95 \% \mathrm{CL}$ & \\
\hline HH size & 1.147 & 1.146 & 1.148 & 1.016 & 1.016 & 1.016 & 1.002 & 1.001 & 1.002 \\
\hline MSA & 1.363 & 1.357 & 1.368 & 1.024 & 1.022 & 1.026 & 1.079 & 1.075 & 1.083 \\
\hline South & 1.165 & 1.159 & 1.172 & 0.854 & 0.853 & 0.855 & 1.055 & 1.049 & 1.061 \\
\hline Poverty & 1.135 & 1.131 & 1.139 & 1.814 & 1.812 & 1.817 & 1.045 & 1.04 & 1.05 \\
\hline Low income & 1.288 & 1.285 & 1.292 & 1.104 & 1.102 & 1.105 & 1.145 & 1.141 & 1.148 \\
\hline Female & 1.428 & 1.425 & 1.432 & 1.816 & 1.814 & 1.817 & 1.9 & 1.895 & 1.905 \\
\hline Age & 0.988 & 0.988 & 0.988 & 0.974 & 0.974 & 0.974 & 0.989 & 0.989 & 0.989 \\
\hline Week & 1.041 & 1.041 & 1.042 & 1.027 & 1.027 & 1.028 & 1.023 & 1.023 & 1.024 \\
\hline $\log$ COVID-19 deaths & 0.675 & 0.673 & 0.677 & 1.049 & 1.048 & 1.05 & 1.01 & 1.007 & 1.012 \\
\hline logPolice killings & 1.644 & 1.639 & 1.649 & 1.045 & 1.044 & 1.046 & 1.394 & 1.389 & 1.399 \\
\hline Lost Job & 1.771 & 1.766 & 1.776 & 1.388 & 1.387 & 1.389 & 1.402 & 1.397 & 1.406 \\
\hline Expect to lose job & 1.276 & 1.273 & 1.28 & 1.872 & 1.87 & 1.874 & 1.847 & 1.841 & 1.853 \\
\hline May 29, 2020 & 1.452 & 1.438 & 1.466 & 0.973 & 0.972 & 0.975 & 1.124 & 1.117 & 1.131 \\
\hline
\end{tabular}

Significant at $95 \%$ confidence level

Dependent variable: frequency of anxiety $(0=$ not at all, $1=$ several days, $2=$ more than half the days, $3=$ nearly every day $)$

Estimates weight by person and household

\section{Robustness Test}

Results from the pooled model are presented in Tables 6 and 7 of Appendix 2. These results are similar in significance and magnitude to those presented above. The estimation of these additional regression frameworks suggests that the findings from this study are robust to various model specifications. 


\section{Discussion}

In the current study, we investigated various factors associated with self-reported levels of anxiety among Black, White, and Hispanic households with and without children during the year 2020. As a universal trend, we found that the frequency of anxiety increased over time for all racial/ ethnic groups. Similarly, a recent or anticipated loss of employment was associated with increased anxiety among all racial/ethnic groups in households with and without children. Among those households containing children, over $50 \%$ of Hispanics and Blacks report that someone in their household has lost their job as a result of the pandemic compared to only $44 \%$ of Whites. This loss of income likely contributed to the higher rates of poverty seen among Hispanic and Black households compared to White households. Although it is clear that members of racial/ethnic minority groups are disproportionately burdened by the economic instability of 2020 , the overall experience of anxiety felt by these groups did not differ based on employment insecurity or financial concerns.

Other studies have shown similar results. Using data from the Census Bureau, Twenge and Joiner (2020) showed that the prevalence of anxiety and depression among US adults was three times higher during the pandemic than a year earlier in Census Bureau-administered national probability samples [54]. Huang and Zhao (2020) showed that despite heightened anxiety levels among all households, the highest levels of anxiety were observed among younger adults - a caveat they attributed to more frequent social media use among this demographic [55]. While their study did not include indicators of social unrest and violence, Jungmann and Witthöft (2020) find that cyberchondria is particularly prevalent among young people whose anxiety is heightened by frequent access to online, virus-related information [56].

While few studies have examined the evolution of anxiety throughout 2020, Brooks et al. (2020) reviewed psychological studies of the initial months of quarantine [57]. They surmised that a variety of negative psychological impacts, including anxiety, could result from stressors including longer quarantine duration, infection fears, frustration, boredom, inadequate supplies, inadequate information, financial loss, and stigma [57]. Having friends, family, and/or acquaintances who either contracted or suspected that they had contracted COVID-19 was a primary indicator of anxiety and depression among college students in China [58]. This proximity to COVID19-related illness and death is also cited for the increase in poor mental health outcomes among healthcare workers [59]. In addition to the workforce sector, a variety of sociodemographic factors have been associated with
COVID-19-related anxiety including household size [58], urbanicity [60], gender [61], education [62], and age [63].

This study is the first, to our knowledge, to extensively examine the effect of notable stressors in 2020, namely, COVID-19 mortality, police violence, and economic and educational disruption on self-reported anxiety among various racial and ethnic groups in the USA. Results showed that, despite higher anxiety levels among all subgroups, notable differences were also found in the specific associations. Household size was inversely related to anxiety among all respondents with no children. Although quarantine mandates during this period of time resulted in forced isolation-a known contributor to anxiety [57], those with larger households may have benefited from the social support of household members during the time they were not able to interact with those outside of the household. This inverse relationship between household size anxiety was also present for Black and White respondents with children, but these variables demonstrated a positive relationship for Hispanics with children. On average, the number of people living in Hispanic households, both with and without children, was greater compared to Black and White households. It is plausible that although having more adult members of a household is a protective factor against anxiety associated with isolation, having a greater number of children in the household could result in greater anxiety. Hispanic households with children also reported less anxiety in relation to urbanicity and low income compared to other racial/ethnic households. A larger proportion of Hispanic households with children, compared to other racial/ethnic households with children, were located in an urban area. For low-income individuals, living in an urban location allows greater access to a wide range of social services [64, 65], such as food pantries, rental assistance, and mental health services that may have lessened the anxiety related to COVID-19 disruptions.

Among households with children, the frequency of anxiety for White respondents was highly responsive to statelevel COVID-19 fatalities, while the association between police-related violence and anxiety was the highest among Black respondents. Previous studies have reported similar racial/ethnic differences in the psychological impact of COVID-19 [25, 66]. In March 2020, Wolf and colleagues found that Blacks were more likely than Whites to report only being "a little worried" or "not worried at all" about contracting COVID-19 [66]. The minimization of the psychological impact of COVID-19 among racial/ethnic minorities appears to be a paradox when considering the higher risk of COVID-19 mortality reported for minority groups [13]. However, the seeming contradiction between the perception of risk and the actual risk is not without precedent. Investigators exploring the racial difference in cancer disparities report heightened fatalistic cancer beliefs and a lower perceived risk of cancer and cancer worries among 
non-Hispanic Blacks despite this group being at an increased actual risk of developing cancer [67]. Similar evidence of an incongruence between perceived and actual risk among racial/ethnic minorities has been documented for other health behaviors and diagnoses [68-72]. Mental health resiliency may also be diminished among racial/ethnic minorities. The theory of Minorities' Diminished Returns [73, 74] suggests that widely accepted protective factors against COVID-19 related distress, such as socioeconomic status, employment security, housing status, education, marital status, and social support may have a weaker protective effect for members of racial/ethnic minority groups [75-81].

In contrast to lower COVID-19-related anxiety, Black Americans experienced more anxiety related to anticipated police encounters compared to Whites [82]. A study using data on 4386 respondents to the Survey of the Health of Urban Residents [83] found that beyond one's personal exposure to police brutality, the anticipation of police violence was related to increased depression and anxiety. During 2020, widespread media coverage of police brutality along with several high-profile police killings could have increased the public fear of police-related violence, this fear being particularly salient for racial and ethnic minorities.

News of George Floyd's death on May 25, 2020, while in the custody of police officers [9], was circulated widely through broadcast coverage and social media platforms [31]. Floyd's death sparked nearly immediate and widespread social unrest. Over subsequent days, demonstrations were organized across the USA. As early as May 31st, the National Guard was deployed in an effort to curb rioting and looting that broke out in some areas $[84,85]$. Week 5 of the HHPS (May 28-June 2, 2020) corresponded closest to the date following the death of George Floyd. Anxiety during this week was elevated in Black and Hispanic households without children, while there was very little change in the anxiety of White households without children, compared to other times during 2020. Among households with children, the frequency of anxiety during this time increased substantially for Black households and was slightly elevated for White households. Notably, anxiety was reduced for Hispanic households with children during the time following Floyd's death.

In the current study, educational disruption was associated with an increased frequency in anxiety but only among Black and White households. Black caregivers who reported a change in educational delivery from traditional, in-person learning to either a distance-learning format or a complete school closure demonstrated a 2.67-fold increase in anxiety compared to households where educational delivery did not change. Likewise, compared to White caregivers with children in the home who did not experience a disruption in education, those who experienced a change in educational delivery reported twice the frequency of anxiety. Various factors may be responsible for the stress of providing educational oversight for children in the home. For caregivers able to continue their employment and work remotely from home, the anxiety of overseeing course work may originate from balancing employment demands [86, 87]. Among caregivers who recently suffered a job loss, lack job flexibility, or are among the multitude of Americans living in poverty, stress may emerge as a result of not being able to provide the resources (e.g., computers, tablets, or Internet access) necessary to support remote learning for the children in their home [46, 88].

\section{Limitations}

While the results presented here are both plausible and logical, this study faced several major limitations. First, the HPS is only a temporary, short-term data collection instrument. Therefore, no historical data exists. Second, the cross-sectional nature of the data made it impossible to determine causality. Each week of the HPS included a different group of respondents making the evolution of individual anxiety impossible to track. While the average number of respondents who reported frequent anxiety increased, this study cannot definitively attribute these increases to the COVID-19 pandemic or the economic manifestations thereof. Third, although important differences were uncovered in the stressors related to anxiety reported among different demographic cohorts, racial and ethnic classifications were self-reported by survey respondents. Given that $2.4 \%$ of the population identifies as multiracial and the number of mixed-race families is steadily increasing, classifying households as a single racial/ethnic group may result in a mischaracterization for some households.

\section{Conclusion}

In summary, the widespread uncertainty of 2020 manifested differently among Black, White, and Hispanic households in the USA. Anxiety levels among all racial and ethnic groups increased as the year progressed. Anxiety related to external factors was likely exacerbated by recent or anticipated job loss that compounded existing economic instability shared by all racial/ethnic groups. Households with children faced added challenges as the educational system forced traditional learning to transition to a home-based model, and parents became responsible for educational outcomes. Among White households, COVID-19 deaths were significantly associated with anxiety while police-related violence and the highly publicized death of George Floyd were associated with anxiety among Black households. While not all households experienced the same associations, 2020 brought societal turmoil, forced isolation, and ambiguous challenges to American households breeding new anxiety and uncertainty for millions. 


\section{Appendix 1}

Table 5 Ordered logistic regression with interaction terms

Ordered logistic regression results for the full sample with interaction terms

\begin{tabular}{|c|c|c|c|c|c|c|}
\hline & \multicolumn{3}{|c|}{ Households with children } & \multicolumn{3}{|c|}{ Household without children } \\
\hline AIC & \multicolumn{3}{|c|}{$133,741,707$} & \multicolumn{3}{|l|}{$301,509,348$} \\
\hline $\mathrm{SC}$ & \multicolumn{3}{|l|}{$133,741,731$} & \multicolumn{3}{|l|}{$301,509,375$} \\
\hline \multirow[t]{2}{*}{$-2 \log L$} & \multicolumn{3}{|l|}{$133,741,701$} & \multicolumn{3}{|l|}{$301,509,342$} \\
\hline & Estimate & Std err & Chi squ & Estimate & Std err & Chi squ \\
\hline Intercept (1) & -2.5858 & 0.00363 & $507,927.9$ & -1.6704 & 0.00191 & $763,593.2$ \\
\hline Intercept (2) & -1.7036 & 0.00362 & $221,785.3$ & -0.8675 & 0.00191 & $207,273.7$ \\
\hline Intercept (3) & $-\mathbf{0 . 0 7 7}$ & 0.00361 & 455.3696 & 0.7107 & 0.00191 & $139,194.7$ \\
\hline HH size & $\mathbf{0 . 0 0 8 3 7}$ & 0.00022 & 1444.17 & 0.011 & 0.000117 & 8808.066 \\
\hline MSA & 0.0822 & 0.00099 & 6897.313 & 0.0646 & 0.000713 & 8226.195 \\
\hline Poverty & 0.4603 & 0.00137 & $113,537.5$ & 0.5759 & 0.000681 & 715,668 \\
\hline Low income & 0.0758 & 0.000707 & $11,496.37$ & 0.1182 & 0.000497 & $56,570.35$ \\
\hline Black & 0.1125 & 0.00248 & 2063.486 & -0.5627 & 0.00139 & $164,796.2$ \\
\hline Hispanic & -0.4054 & 0.003 & $18,258.32$ & 0.0943 & 0.00149 & 3999.568 \\
\hline Female & 0.5573 & 0.000615 & $819,881.8$ & 0.5905 & 0.000378 & $2,435,566$ \\
\hline Age & -0.0155 & 0.000023 & $439,508.8$ & -0.0248 & 0.00001 & $5,761,994$ \\
\hline Week & $\mathbf{0 . 0 3 0 7}$ & 0.000057 & $287,154.2$ & 0.0281 & 0.000036 & $602,357.2$ \\
\hline School change & 0.5676 & 0.00185 & $93,702.2$ & & & \\
\hline $\log$ COVID-19 deaths & 0.00335 & 0.000472 & 50.3815 & 0.0276 & 0.0003 & 8453.845 \\
\hline logPolice killings & 0.1633 & 0.00069 & $55,993.22$ & 0.116 & 0.000468 & $61,465.51$ \\
\hline Lost job & 0.3423 & 0.000621 & 304,175 & 0.3784 & 0.000449 & $711,235.8$ \\
\hline Expect to lose job & 0.6726 & 0.000646 & $1,084,968$ & 0.5763 & 0.000477 & $1,456,975$ \\
\hline South & -0.2626 & 0.000824 & $101,515.6$ & -0.1407 & 0.000545 & $66,756.39$ \\
\hline May 29, 2020 & $\mathbf{0 . 0 5 3 6}$ & 0.00115 & 2181.943 & 0.0296 & 0.00073 & 1647.724 \\
\hline HH size*Black & -0.0621 & 0.000468 & $17,613.61$ & 0.1465 & 0.000313 & $219,274.1$ \\
\hline Poverty*Black & -0.1666 & 0.00252 & 4360.525 & -0.3359 & 0.00184 & $33,204.14$ \\
\hline Low income*Black & 0.000878 & 0.00174 & 0.2532 & -0.0127 & 0.00156 & 65.9702 \\
\hline Female*Black & -0.3148 & 0.00151 & $43,542.12$ & -0.162 & 0.00124 & $17,184.47$ \\
\hline HH size*Hispanic & 0.0662 & 0.000536 & $15,264.48$ & -0.0209 & 0.000401 & 2719.442 \\
\hline Poverty*Hispanic & -0.3594 & 0.00331 & $11,766.4$ & -0.6291 & 0.00236 & $71,325.77$ \\
\hline Low income*Hispanic & -0.1894 & 0.00181 & $10,958.93$ & 0.0553 & 0.00175 & 995.4539 \\
\hline Female*Hispanic & 0.0971 & 0.00164 & 3502.382 & 0.0782 & 0.00141 & 3058.664 \\
\hline \multicolumn{7}{|l|}{ Odds ratio estimates } \\
\hline & Estimate & $95 \% \mathrm{CL}$ & & Estimate & $95 \% \mathrm{CL}$ & \\
\hline HH size & 0.985 & 0.985 & 0.985 & 0.976 & 0.975 & 0.976 \\
\hline MSA & 1.006 & 1.006 & 1.006 & 1.023 & 1.022 & 1.023 \\
\hline Poverty & 1.086 & 1.084 & 1.088 & 1.067 & 1.065 & 1.068 \\
\hline Low income & 1.48 & 1.476 & 1.483 & 1.66 & 1.658 & 1.662 \\
\hline Female & 1.055 & 1.054 & 1.057 & 1.128 & 1.127 & 1.129 \\
\hline Age & 0.693 & 0.692 & 0.694 & 0.735 & 0.734 & 0.736 \\
\hline Week & 0.86 & 0.858 & 0.861 & 1.006 & 1.005 & 1.008 \\
\hline Black & 1.679 & 1.677 & 1.681 & 1.789 & 1.787 & 1.79 \\
\hline Hispanic & 1.031 & 1.031 & 1.031 & 1.029 & 1.028 & 1.029 \\
\hline School change & 1.764 & 1.758 & 1.77 & & & \\
\hline $\log$ COVID-19 deaths & 1.003 & 1.002 & 1.004 & 1.028 & 1.027 & 1.029 \\
\hline
\end{tabular}


Table 5 continued

Ordered logistic regression results for the full sample with interaction terms

\begin{tabular}{|c|c|c|c|c|c|c|}
\hline logPolice killings & 1.177 & 1.176 & 1.179 & 1.123 & 1.122 & 1.124 \\
\hline Lost job & 1.408 & 1.406 & 1.41 & 1.46 & 1.459 & 1.461 \\
\hline Expect to lose job & 1.959 & 1.957 & 1.962 & 1.779 & 1.778 & 1.781 \\
\hline South & 0.769 & 0.768 & 0.77 & 0.869 & 0.868 & 0.87 \\
\hline May 29, 2020 & 1.055 & 1.053 & 1.057 & 1.03 & 1.029 & 1.032 \\
\hline
\end{tabular}

Significant at $95 \%$ confidence level.

Dependent variable: frequency of anxiety $(0=$ not at all, $1=$ several days, $2=$ more than half the days, $3=$ nearly every day $)$.

Estimates weighted by person and household. 


\section{Appendix 2}

Table 6 Regression results for the pooled model for households without children

Ordered logistic regression for households without children

\begin{tabular}{|c|c|c|c|c|c|c|c|c|c|}
\hline AIC & $282,804,329$ & & & & & & & & \\
\hline $\mathrm{SC}$ & $282,804,596$ & & & & & & & & \\
\hline \multirow[t]{2}{*}{$-2 \log L$} & $282,804,269$ & & & & & & & & \\
\hline & Estimate & Std err & Chi squ & & & & & & \\
\hline Intercept (3) & -1.777 & 0.00198 & $804,086.6$ & & & & & & \\
\hline Intercept (2) & -0.9751 & 0.00198 & $243,616.1$ & & & & & & \\
\hline Intercept (1) & 0.6016 & 0.00197 & $92,848.87$ & & & & & & \\
\hline HH size & 0.0288 & 0.000105 & $75,552.92$ & & & & & & \\
\hline MSA & 0.0631 & 0.000721 & 7666.786 & & & & & & \\
\hline Poverty & 0.4894 & 0.000617 & 629,120 & & & & & & \\
\hline Low income & 0.1085 & 0.000462 & $55,083.14$ & & & & & & \\
\hline Black & 2.0526 & 0.00953 & $46,408.23$ & & & & & & \\
\hline Hispanic & 0.2573 & 0.00776 & 1099.345 & & & & & & \\
\hline Female & 0.5778 & 0.000351 & $2,714,389$ & & & & & & \\
\hline Age & -0.0248 & 0.00001 & $5,744,262$ & & & & & & \\
\hline Week & 0.0279 & 0.000036 & $591,957.1$ & Black* $\chi$ & & & Hispanic $*$ & & \\
\hline logCOVID-19 deaths & 0.0439 & 0.000313 & $19,648.09$ & -0.3951 & 0.00152 & $67,385.47$ & -0.0602 & 0.00126 & 2264.636 \\
\hline logPolice killings & 0.0484 & 0.000507 & 9126.457 & 0.4579 & 0.0015 & $93,472.45$ & 0.3465 & 0.00173 & $39,970.53$ \\
\hline Lost job & 0.3678 & 0.000488 & $567,254.3$ & 0.212 & 0.00149 & $20,310.04$ & -0.0769 & 0.00169 & 2062.109 \\
\hline Expect to lose job & 0.6133 & 0.000527 & $1,352,291$ & -0.3787 & 0.00152 & $62,485.09$ & 0.00808 & 0.00174 & 21.4946 \\
\hline South & -0.158 & 0.000564 & $78,422.57$ & 0.1738 & 0.00279 & 3877.742 & 0.2898 & 0.00294 & 9709.859 \\
\hline May 29, 2020 & 0.0123 & 0.000759 & 261.1555 & 0.2413 & 0.00495 & 2373.591 & 0.0818 & 0.00321 & 648.1159 \\
\hline \multicolumn{10}{|l|}{ Odds ratio estimates } \\
\hline & Estimate & $95 \% \mathrm{CL}$ & & & & & & & \\
\hline HH Sie & 1.029 & 1.029 & 1.029 & & & & & & \\
\hline MSA & 1.065 & 1.064 & 1.067 & & & & & & \\
\hline Poverty & 1.631 & 1.629 & 1.633 & & & & & & \\
\hline Low income & 1.115 & 1.114 & 1.116 & & & & & & \\
\hline Female & 1.782 & 1.781 & 1.783 & & & & & & \\
\hline Age & 0.976 & 0.976 & 0.976 & & & & & & \\
\hline Week & 1.028 & 1.028 & 1.028 & & & & & & \\
\hline Black & 0.796 & 0.795 & 0.797 & & & & & & \\
\hline Hispanic & 0.999 & 0.997 & 1.000 & & & & & & \\
\hline logCOVID-19 deaths & 1.006 & 1.005 & 1.007 & & & & & & \\
\hline logPolice killings & 1.116 & 1.115 & 1.117 & & & & & & \\
\hline Lost job & 1.464 & 1.463 & 1.465 & & & & & & \\
\hline Expect to lose Job & 1.788 & 1.786 & 1.79 & & & & & & \\
\hline South & 0.883 & 0.882 & 0.884 & & & & & & \\
\hline May 29, 2020 & 1.039 & 1.037 & 1.041 & & & & & & \\
\hline
\end{tabular}

Significant at $95 \%$ confidence level.

Dependent variable: frequency of anxiety $(0=$ not at all, $1=$ several days, $2=$ more than half the days, $3=$ nearly every day).

Estimates weighted by person and household. 
Table 7 Regression results for pooled model for households with children

Ordered logistic regression model for households with children

\begin{tabular}{|c|c|c|c|c|c|c|c|c|c|}
\hline AIC & $128,135,505$ & & & & & & & & \\
\hline $\mathrm{SC}$ & $128,135,773$ & & & & & & & & \\
\hline \multirow[t]{2}{*}{$-2 \log L$} & $128,135,439$ & & & & & & & & \\
\hline & Estimate & Std err & Chi squ & & & & & & \\
\hline Intercept (1) & -2.8807 & 0.00383 & $565,593.9$ & & & & & & \\
\hline Intercept (2) & -1.9973 & 0.00382 & $273,456.8$ & & & & & & \\
\hline Intercept (3) & -0.369 & 0.00381 & 9385.687 & & & & & & \\
\hline HH size & 0.0104 & 0.000187 & 3111.327 & & & & & & \\
\hline MSA & 0.0942 & 0.00101 & 8707.795 & & & & & & \\
\hline Poverty & 0.4146 & 0.00111 & $139,175.9$ & & & & & & \\
\hline Low income & 0.0245 & 0.00063 & 1504.411 & & & & & & \\
\hline Black & 0.8874 & 0.014 & 4023.925 & & & & & & \\
\hline Hispanic & 2.5965 & 0.0116 & 49,871 & & & & & & \\
\hline Female & 0.5322 & 0.000536 & $984,507.3$ & & & & & & \\
\hline Age & -0.0155 & 0.000023 & 436,415 & & & & & & \\
\hline Week & 0.0314 & 0.000058 & $297,755.7$ & Black $^{*} \chi$ & & & Hispanic $^{*} \chi$ & & \\
\hline School change & 0.6113 & 0.002 & $93,328.8$ & 0.3348 & 0.009 & 1382.025 & -0.5612 & 0.00599 & 8773.066 \\
\hline $\log$ COVID-19 deaths & 0.0436 & 0.000506 & 7434.326 & $-\mathbf{0 . 2 3 7 7}$ & 0.00173 & $18,967.52$ & -0.3473 & 0.00161 & $46,580.8$ \\
\hline logPolice killings & 0.1617 & 0.000805 & $40,399.23$ & 0.0594 & 0.00174 & 1157.474 & 0.0502 & 0.00203 & 613.0939 \\
\hline Lost job & 0.3539 & 0.000717 & $243,774.5$ & -0.0651 & 0.00167 & 1509.484 & -0.0164 & 0.00203 & 65.135 \\
\hline Expect to lose job & 0.7182 & 0.000758 & $898,531.9$ & -0.2876 & 0.00168 & $29,283.91$ & 0.0037 & 0.00199 & 3.4581 \\
\hline South & -0.2655 & 0.000894 & $88,209.26$ & 0.1669 & 0.00306 & 2981.212 & -0.2332 & 0.00284 & 6730.196 \\
\hline May 29, 202 & 0.1113 & 0.00127 & 7692.278 & 0.3611 & 0.00403 & 8037.985 & -0.9089 & 0.00398 & $52,020.87$ \\
\hline \multicolumn{10}{|l|}{ Odds ratio estimates } \\
\hline & Estimate & $95 \% \mathrm{CL}$ & & & & & & & \\
\hline HH size & 1.01 & 1.01 & 1.011 & & & & & & \\
\hline MSA & 1.099 & 1.097 & 1.101 & & & & & & \\
\hline Poverty & 1.514 & 1.51 & 1.517 & & & & & & \\
\hline Low income & 1.025 & 1.023 & 1.026 & & & & & & \\
\hline Female & 1.703 & 1.701 & 1.704 & & & & & & \\
\hline Age & 0.985 & 0.985 & 0.985 & & & & & & \\
\hline Week & 1.032 & 1.032 & 1.032 & & & & & & \\
\hline Black & 0.72 & 0.719 & 0.721 & & & & & & \\
\hline Hispanic & 0.848 & 0.846 & 0.849 & & & & & & \\
\hline School change & 1.822 & 1.814 & 1.829 & & & & & & \\
\hline $\log$ COVID-19 deaths & 0.966 & 0.965 & 0.967 & & & & & & \\
\hline logPolice killings & 1.194 & 1.192 & 1.195 & & & & & & \\
\hline Lost job & 1.407 & 1.405 & 1.409 & & & & & & \\
\hline Expect to lose job & 1.959 & 1.957 & 1.962 & & & & & & \\
\hline South & 0.767 & 0.765 & 0.768 & & & & & & \\
\hline May 29, 2020 & 1.066 & 1.063 & 1.068 & & & & & & \\
\hline
\end{tabular}

Significant at $95 \%$ confidence level.

Dependent variable: frequency of anxiety $(0=$ not at all, $1=$ several days, $2=$ more than half the days, $3=$ nearly every day).

Estimates weighted by person and household. 
Data Availability Data are publicly available from sources provided in the manuscript.

Code Availability Not applicable.

\section{Declarations}

Competing Interests The authors declare no competing interests.

\section{References}

1. Caggiano G, Castelnuovo E, Kima R. The global effects of COVID-19-induced uncertainty. Economics Letters. 2020;194:109392.

2. Altig D, Baker S, Barrero JM, Bloom N, Bunn P, Chen S, et al. Economic uncertainty before and during the COVID-19 pandemic. Journal of Public Economics. 2020;191:104274.

3. Baker SR, Bloom N, Davis SJ, Terry SJ. COVID-induced economic uncertainty [Internet]. National Bureau of Economic Research. 2020. Report No.: 26983. Available from: https://www. nber.org/papers/w26983. Accessed 6 Aug 2021.

4. Dubey S, Biswas P, Ghosh R, Chatterjee S, Dubey MJ, Chatterjee $\mathrm{S}$, et al. Psychosocial impact of COVID-19. Diabetes \& Metab Syndr. 2020;14:779-88.

5. Godinić D, Obrenovic B, Hudaykulov A. Effects of economic uncertainty on mental health in the COVID-19 pandemic context: social identity disturbance, job uncertainty and psychological well-being model. Int J Innov Econ. 2020;6:61-74.

6. Hisaka A, Yoshioka H, Hatakeyama H, Sato H, Onouchi Y, Anzai $\mathrm{N}$. Global comparison of changes in the number of test-positive cases and deaths by coronavirus infection (COVID-19) in the world. J Clin Med. 2020;9:1904.

7. Bruine de Bruin W, Saw H-W, Goldman DP. Political polarization in US residents' COVID-19 risk perceptions, policy preferences, and protective behaviors. J Risk Uncertain. 2020;61:177-94.

8. Galea S, Abdalla SM. COVID-19 pandemic, unemployment, and civil unrest: underlying deep racial and socioeconomic divides. JAMA. 2020;324:227-8.

9. Hill E, Tiefenthäler A, Triebert C, Jordan D, Willis H, Stein R. How George Floyd was killed in police custody. The New York Times [Internet]. 2020 Jun 1 [cited 2021 Aug 6]; Available from: https://www.nytimes.com/2020/05/31/us/george-floyd-investigat ion.html

10. Adhikari S, Pantaleo NP, Feldman JM, Ogedegbe O, Thorpe L, Troxel AB. Assessment of community-level disparities in coronavirus disease 2019 (COVID-19) infections and deaths in large US metropolitan areas. JAMA Netw Open. 2020;3:e2016938-e2016938.

11. Couch KA, Fairlie RW, Xu H. Early evidence of the impacts of COVID-19 on minority unemployment. Journal of Public Economics. 2020;192:104287.

12. Gonzalez D, Karpman M, Kenney GM, Zuckerman S. Hispanic adults in families with noncitizens disproportionately feel the economic fallout from COVID-19 [Internet]. Urban Institute. 2020 [cited 2021 Aug 6]. Available from: https://www.urban. org/research/publication/hispanic-adults-families-noncitizensdisproportionately-feel-economic-fallout-covid-19

13. Gross CP, Essien UR, Pasha S, Gross JR, Wang S, Nunez-Smith M. Racial and ethnic disparities in population-level COVID-19 mortality. J GEN INTERN MED. 2020;35:3097-9.
14. Kouzy R, Jaoude JA, Kraitem A, Alam MBE, Karam B, Adib E, et al. Coronavirus goes viral: quantifying the COVID-19 misinformation epidemic on Twitter. Cureus [Internet]. Cureus; 2020 [cited 2021 Aug 6];12. Available from: https://www.cureus. com/articles/28976-coronavirus-goes-viral-quantifying-thecovid-19-misinformation-epidemic-on-twitter

15. Tagliabue F, Galassi L, Mariani P. The, "pandemic" of disinformation in COVID-19. SN Compr Clin Med. 2020;2:1287-9.

16. Carrion-Alvarez D, Tijerina-Salina PX. Fake news in COVID19: a perspective Health Promot Perspect. Tabriz University of Medical Sciences. 2020;10:290-1.

17. Fleming N. Coronavirus misinformation, and how scientists can help to fight it. Nature. 2020;583:155-6.

18. Patel AB, Verma A. COVID-19 and angiotensin-converting enzyme inhibitors and angiotensin receptor blockers: what is the evidence? JAMA. 2020;323:1769-70.

19. Alonzi S, La Torre A, Silverstein MW. The psychological impact of preexisting mental and physical health conditions during the COVID-19 pandemic. Psychol Trauma. 2020;12:S236-8.

20. Asmundson GJG, Paluszek MM, Landry CA, Rachor GS, McKay D, Taylor S. Do pre-existing anxiety-related and mood disorders differentially impact COVID-19 stress responses and coping? Journal of Anxiety Disorders. 2020;74:102271.

21. Hodžić N, Hasanović M, Pajević I. COVID-19 affected mental health of at-risk groups of psychiatric patients: two case reports. Psychiatria Danubina [Internet]. Medicinska Naklada d.o.o.; 2020 [cited 2021 Aug 6]; Available from: https://www.scien ceopen.com/document?vid=b1fa23e $8-075 d-44 d c-9 b 6 f-9 b c 87$ $9758 \mathrm{~d} 39$

22. Liu CH, Erdei C, Mittal L. Risk factors for depression, anxiety, and PTSD symptoms in perinatal women during the COVID-19 pandemic. Psychiatry Research. 2021;295:113552.

23. Pinkham AE, Ackerman RA, Depp CA, Harvey PD, Moore RC. A longitudinal investigation of the effects of the COVID-19 pandemic on the mental health of individuals with pre-existing severe mental illnesses. Psychiatry Research. 2020;294:113493.

24 Wong SYS, Zhang D, Sit RWS, Yip BHK, Chung RY, Wong CKM, et al. Impact of COVID-19 on loneliness, mental health, and health service utilisation: a prospective cohort study of older adults with multimorbidity in primary care. Br J Gen Pract. British Journal of General Practice. 2020;70:e817-24.

25. Holingue C, Badillo-Goicoechea E, Riehm KE, Veldhuis CB, Thrul J, Johnson RM, et al. Mental distress during the COVID-19 pandemic among US adults without a pre-existing mental health condition: findings from American trend panel survey. Preventive Medicine. 2020;139:106231.

26. Ettman CK, Abdalla SM, Cohen GH, Sampson L, Vivier PM, Galea S. Low assets and financial stressors associated with higher depression during COVID-19 in a nationally representative sample of US adults. J Epidemiol Community Health. BMJ Publishing Group Ltd. 2020;75:501-8.

27. Le K, Nguyen M. The psychological burden of the COVID-19 pandemic severity. Economics \& Human Biology. 2021;41:100979.

28. Liu CH, Zhang E, Wong GTF, Hyun S, Chris Hahm H. Factors associated with depression, anxiety, and PTSD symptomatology during the COVID-19 pandemic: clinical implications for U.S. young adult mental health. Psychiatry Research. 2020;290:113172.

29. Novotný JS, Gonzalez-Rivas JP, Kunzová Š, Skladaná M, Pospíšilová A, Polcrová A, et al. Risk factors underlying COVID19 lockdown-induced mental distress. Front Psychiatry [Internet]. Frontiers; 2020 [cited 2021 Aug 6];0. Available from: https://doi. org/10.3389/fpsyt.2020.603014/full

30. Ruffolo M, Price D, Schoultz M, Leung J, Bonsaksen T, Thygesen $\mathrm{H}$, et al. Employment uncertainty and mental health during the 
COVID-19 pandemic initial social distancing implementation: a cross-national study. Glob Soc Welf. 2021;8:141-50.

31. Buchanan L, Bui Q, Patel JK. Black Lives Matter may be the largest movement in U.S. history. The New York Times [Internet]. 2020 Jul 3 [cited 2021 Aug 6]; Available from: https:// www.nytimes.com/interactive/2020/07/03/us/george-floyd-prote sts-crowd-size.html

32. Thomas T, Gabbatt A, Barr C. Nearly 1,000 instances of police brutality recorded in US anti-racism protests [Internet]. The Guardian. 2020 [cited 2021 Aug 6]. Available from: http://www. theguardian.com/us-news/2020/oct/29/us-police-brutality-protest

33. Ross CT. A multi-level Bayesian analysis of racial bias in police shootings at the county-level in the United States, 2011-2014. PLOS ONE Public Library of Science. 2015;10:e0141854.

34 Edwards F, Esposito MH, Lee H. Risk of Police-involved death by race/ethnicity and place, United States, 2012-2018. Am J Public Health. American Public Health Association; 2018;108:1241-8.

35. Ang D. The effects of police violence on inner-city students*. Q J Econ. 2021;136:115-68.

36. Legewie J, Fagan J. Aggressive policing and the educational performance of minority youth. Am Sociol Rev SAGE Publications Inc. 2019;84:220-47.

37. UNESCO. From COVID-19 learning disruption to recovery: a snapshot of UNESCO's work in education in 2020 [Internet]. UNESCO. 2020 [cited 2021 Aug 6]. Available from: https://en. unesco.org/news/covid-19-learning-disruption-recovery-snapshotunescos-work-education-2020

38. UNESCO. UNESCO figures show two thirds of an academic year lost on average worldwide due to COVID-19 school closures [Internet]. UNESCO. 2021 [cited 2021 Aug 6]. Available from: https://en.unesco.org/news/unesco-figures-show-two-thirds-acade mic-year-lost-average-worldwide-due-covid-19-school

39. McNeill L, Puleo E, Bennett G, Emmons K. Exploring social contextual correlates of computer ownership and frequency of use among urban, low-income, public housing adult residents. Journal of Medical Internet Research. 2007;9:e953.

40. Pearson T. A technology crisis facing minority students. TECHTRENDS TECH TRENDS. 2002;46:15-20.

41. Fairlie R. Race and the digital divide. eScholarship [Internet]. 2014 [cited 2021 Aug 6]; Available from: https://escholarship. org/uc/item/48h8h99w

42. FCC. 2020 Broadband Deployment Report [Internet]. Federal Communications Commission. 2020 [cited 2021 Aug 6]. Available from: https://www.fcc.gov/reports-research/reports/broad band-progress-reports/2020-broadband-deployment-report

43. Jones S, Johnson-Yale C, Millermaier S, Pérez FSUS. college students' internet use: race, gender and digital divides. J ComputMediat Commun. 2009;14:244-64.

44. Prieger J. The broadband digital divide and the economic benefits of mobile broadband for rural areas. Telecommunications Policy Elsevier. 2013;37:483-502.

45. APA. Stress in America ${ }^{\mathrm{TM}}$ 2020: stress in the time of COVID19, Volume One [Internet]. American Psychological Association. 2020 [cited 2021 Aug 10]. Available from: https://www.apa.org/ news/press/releases/stress/2020/report

46. Lee SJ, Ward KP, Chang OD, Downing KM. Parenting activities and the transition to home-based education during the COVID-19 pandemic. Child Youth Serv Rev. 2021;122:105585.

47. Parolin, Zachary, Megan A. Curran, Jordan Matsudaira, Jane Waldfogel and Christopher Wimer. 2020. "Monthly Poverty Rates in the United States during the COVID-19 Pandemic." Poverty and Social Policy Discussion Paper. New York, NY: Center on Poverty and Social Policy.

48. Shah SMA, Mohammad D, Qureshi MFH, Abbas MZ, Aleem S. Prevalence, psychological responses and associated correlates of depression, anxiety and stress in a global population, during the coronavirus disease (COVID-19) pandemic. Community Ment Health J. 2021;57:101-10.

49. Beggs JJ. The institutional environment: implications for race and gender inequality in the U.S. labor market American Sociological Review. [American Sociological Association, Sage Publications, Inc]. 1995;60:612-33.

50. Elliott JR, Smith RA. Race, gender, and workplace power. Am Sociol Rev SAGE Publications Inc. 2004;69:365-86.

51. Chaudry A, Wimer C, Macartney S, Frohlich L, Campbell C, Swenson K, et al. Poverty in the United States: 50-year trends and safety net impacts. Office of the Assistant Secretary for Planning and Evaluation. 2016; PDF file available at https://alliancetoendhu nger.org/wp-content/uploads/2018/03/50YearTrends.pdf

52. Woolf SH, Johnson RE, Geiger HJ. The rising prevalence of severe poverty in America: a growing threat to public health. American Journal of Preventive Medicine Elsevier. 2006;31:332-341.e2.

53. US Census Bureau. Household Pulse Survey Data Tables [Internet]. Census.gov. 2021 [cited 2021 Aug 11]. Available from: https://www.census.gov/programs-surveys/household-pulse-survey/data.html

54. Twenge JM, Joiner TEUS. Census Bureau-assessed prevalence of anxiety and depressive symptoms in 2019 and during the 2020 COVID-19 pandemic. Depress Anxiety. 2020;37:954-6.

55. Huang Y, Zhao N. Generalized anxiety disorder, depressive symptoms and sleep quality during COVID-19 outbreak in China: a web-based cross-sectional survey. Psychiatry Res. 2020;288:112954.

56. Jungmann SM, Witthöft M. Health anxiety, cyberchondria, and coping in the current COVID-19 pandemic: which factors are related to coronavirus anxiety? J Anxiety Disord. 2020;73:102239.

57. Brooks SK, Webster RK, Smith LE, Woodland L, Wessely S, Greenberg N, et al. The psychological impact of quarantine and how to reduce it: rapid review of the evidence. Lancet. 2020;395:912-20.

58. Cao W, Fang Z, Hou G, Han M, Xu X, Dong J, et al. The psychological impact of the COVID-19 epidemic on college students in China. Psychiatry Res. 2020;287:112934.

59. Lu W, Wang H, Lin Y, Li L. Psychological status of medical workforce during the COVID-19 pandemic: a cross-sectional study. Psychiatry Res. 2020;288:112936.

60. Özdin S, BayrakÖzdin Ş. Levels and predictors of anxiety, depression and health anxiety during COVID-19 pandemic in Turkish society: the importance of gender. Int J Soc Psychiatry SAGE Publications Ltd. 2020;66:504-11.

61. Zhang J, Lu H, Zeng H, Zhang S, Du Q, Jiang T, et al. The differential psychological distress of populations affected by the COVID-19 pandemic. Brain Behav Immun. 2020;87:49-50.

62. Du J, Dong L, Wang T, Yuan C, Fu R, Zhang L, et al. Psychological symptoms among frontline healthcare workers during COVID19 outbreak in Wuhan. Gen Hosp Psychiatry. 2020;67:144-5.

63. Mazza C, Ricci E, Biondi S, Colasanti M, Ferracuti S, Napoli C, et al. A nationwide survey of psychological distress among Italian people during the COVID-19 pandemic: immediate psychological responses and associated factors. Int J Environ Res Public Health. 2020;17:E3165.

64. Vlahov D. Urbanization, Urbanicity, and Health. Journal of Urban Health: Bulletin of the New York Academy of Medicine. 2002;79:1S - 12 .

65. Allard SW. Mismatches and unmet need: access to social services in urban and rural America. In: Ziliak JP, editor. Welfare Reform and Its Long-Term Consequences for America's Poor [Internet]. Cambridge: Cambridge University Press; 2009 [cited 2021 Nov 4]. p. 337-68. Available from: https://www.cambridge.org/core/ product/identifier/CBO9780511605383A017/type/book_part

66. Wolf MS, Serper M, Opsasnick L, O'Conor RM, Curtis L, Benavente JY, et al. Awareness, attitudes, and actions related to 
COVID-19 among adults with chronic conditions at the onset of the U.S. outbreak. Ann Intern Med American College of Physicians. 2020;173:100-9.

67. Assari S, Khoshpouri P, Chalian H. Combined effects of race and socioeconomic status on cancer beliefs, cognitions, and emotions. Healthcare. 2019;7:17.

68. Chalian H, Khoshpouri P, Assari S. Patients' age and discussion with doctors about lung cancer screening: diminished returns of Blacks. AGING MEDICINE. 2019;2:35-41.

69. Waterman AD, Browne T, Waterman BM, Gladstone EH, Hostetter T. Attitudes and behaviors of African Americans regarding early detection of kidney disease. Am J Kidney Dis. 2008;51:554-62.

70. Graham GN, Leath B, Payne K, Guendelman M, Reynolds G, Kim $\mathrm{S}$, et al. Perceived versus actual risk for hypertension and diabetes in the African American community. health promotion practice. SAGE Publications. 2006;7:34-46.

71. Bloom JR, Stewart SL, Oakley-Girvans I, Banks PJ, Chang S. Family history, perceived risk, and prostate cancer screening among African American men. Cancer Epidemiol Biomarkers Prev. American Association for Cancer Research. 2006;15:2167-73.

72. Diaz VA, Mainous AG, Williamson D, Johnson SP, Knoll ME. Cardiovascular and diabetes risk perception in a Hispanic community sample. Ethn Dis. 2012;22:5-11.

73. Assari S. Health disparities due to diminished return among Black Americans: public policy solutions: health disparities due to diminished return among Black Americans. Soc Issues Policy Rev. 2018;12:112-45.

74. Assari S. Unequal gain of equal resources across racial groups. Int J Health Policy Manag. 2017;7:1-9.

75. Wang Y, Kala MP, Jafar TH. Factors associated with psychological distress during the coronavirus disease 2019 (COVID-19) pandemic on the predominantly general population: a systematic review and meta-analysis. PLoS One. 2020;15:e0244630.

76. Kunzler AM, Röthke N, Günthner L, Stoffers-Winterling J, Tüscher $\mathrm{O}$, Coenen $\mathrm{M}$, et al. Mental burden and its risk and protective factors during the early phase of the SARS-CoV-2 pandemic: systematic review and meta-analyses. Global Health. 2021;17:34.

77. Xiong J, Lipsitz O, Nasri F, Lui LMW, Gill H, Phan L, et al. Impact of COVID-19 pandemic on mental health in the general population: a systematic review. J Affect Disord. 2020;277:55-64.

78. Fernández RS, Crivelli L, Guimet NM, Allegri RF, Pedreira ME. Psychological distress associated with COVID-19 quarantine: latent profile analysis, outcome prediction and mediation analysis. J Affect Disord. 2020;277:75-84.
79. Bushman G, Mehdipanah R. J Housing and health inequities during COVID-19: findings from the national Household Pulse Survey. Epidemiol Community Health. 2021. https://doi.org/10.1136/ jech-2021-216764

80. Linton SL, Leifheit KM, McGinty EE, Barry CL, Pollack CE. Association between housing insecurity, psychological distress, and self-rated health among US adults during the COVID-19 pandemic. JAMA Network Open. 2021;4:e2127772.

81. Yu H, Li M, Li Z, Xiang W, Yuan Y, Liu Y, et al. Coping style, social support and psychological distress in the general Chinese population in the early stages of the COVID-19 epidemic. BMC Psychiatry. 2020;20:426.

82. Najdowski CJ, Bottoms BL, Goff PA. Stereotype threat and racial differences in citizens' experiences of police encounters. Law and Human Behavior. US: Educational Publishing Foundation. 2015;39:463-77.

83. Alang S, McAlpine D, McClain M. Police encounters as stressors: associations with depression and anxiety across race. Socius SAGE Publications. 2021;7:2378023121998128.

84. Burch ADS, Cai W, Gianordoli G, McCarthy M, Patel JK. How Black Lives Matter reached every corner of America. The New York Times [Internet]. 2020 Jun 13 [cited 2021 Aug 11]; Available from: https://www.nytimes.com/interactive/2020/06/13/us/ george-floyd-protests-cities-photos.html

85. Cable News Network. In pictures: a racial reckoning in America [Internet]. CNN. 2020 [cited 2021 Aug 11]. Available from: https://www.cnn.com/2020/05/27/us/gallery/george-floyd-demon strations/index.html

86. Chu KA, Schwartz C, Towner E, Kasparian N, Callaghan B. Parenting under pressure: a mixed-methods investigation of the impact of COVID-19 on family life [Internet]. Rochester, NY: Social Science Research Network; 2021 Jan. Report No.: ID 3741254. Available from: https://papers.ssrn.com/abstract=37412 54. Accessed 11 Aug 2021.

87. Heggeness ML. Estimating the immediate impact of the COVID19 shock on parental attachment to the labor market and the double bind of mothers. Rev Econ Household. 2020;18:1053-78.

88. Garbe A, Ogurlu U, Logan N, Cook P. COVID-19 and remote learning: experiences of parents with children during the pandemic. AM J QUALITATIVE RES HA Publication. 2020;4:45-65.

Publisher's Note Springer Nature remains neutral with regard to jurisdictional claims in published maps and institutional affiliations. 